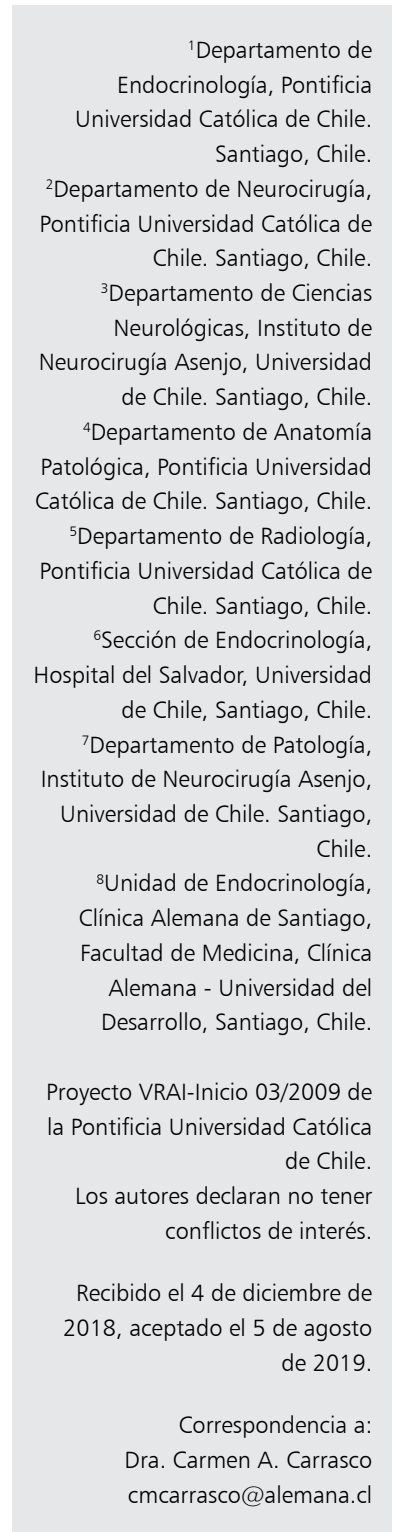

\section{Caracterización de tumores secretores de hormona de crecimiento de acuerdo al patrón granular y su rol en el pronóstico}

\author{
FELIPE VALENZUELA ${ }^{1}$, PABLO VILLANUEVA ${ }^{2}$, \\ DAVID ROJAS Z. ${ }^{3}$, ROGER GEJMAN ${ }^{4}$, ISIDRO HUETE ${ }^{5}$, \\ ROMINA ZUNINO ${ }^{5}$, RENÉ E. DÍAZ ${ }^{6}$, NELSON WOHLLK ${ }^{6}$, \\ CLAUDIA TISSERA ${ }^{7}$, CARMEN A. CARRASCO ${ }^{8}$

\section{Prognostic value of granular pattern of growth hormone secreting tumors}

Background: Classification of growth hormone (GH) - secreting tumors by the granular pattern might predict their clinical behavior in acromegalic patients. There are several other prognostic factors. Aim: To compare the features at presentation and cure rates of patients with GH secreting tumors according to the granular pattern, and to define independent prognostic factors for surgical treatment in these patients. Material and Methods: A retrospective, observational study of 85 active acromegalic patients surgically treated in two medical centers. Results: Seventy-four patients (87\%) were classified as having densely granulated (DG) and 11 (13\%) as sparsely granulated (SG) tumors. The latter were less active biochemically, had a higher rate of macroadenoma and cavernous sinus invasion and had a lower rate of biochemical cure than the DG group. Several characteristics were associated with disease persistence but only age $(\mathrm{Odds}$ ratio $(O R)=0.93)$ and cavernous sinus invasion $(O R=21.7)$ were independently associated in the logistic regression model. Conclusions: The sparsely granulated pattern is associated with a more aggressive behavior, but the main determinants of prognosis are age and cavernous sinus invasion.

(Rev Med Chile 2019; 147: 852-859)

Key words: Acromegaly; Growth Hormone-Secreting Pituitary Adenoma; Immunohistochemistry; Pathology.
L

os tumores somatotropos son tumores productores de hormona del crecimiento $(\mathrm{GH})$ te ${ }^{1}$ y más de $95 \%$ de los casos de acromegalia.

Estos tumores se clasifican, de acuerdo al patrón de gránulos de $\mathrm{GH}$, en densamente granulares (DG) y escasamente granulares $(\mathrm{EG})^{2}$. En el pasado esta distinción se hacía usando la microscopía electrónica, pero en la actualidad se realiza a través de la distribución de filamentos de citoqueratina por inmunohistoquímica en la microscopía de luz $z^{3}$. Con esta técnica, los tumores EG poseen un agregado de filamentos de citoqueratina intracitoplasmáticos llamado "cuerpo fibroso", caracterizado por un patrón de tinción en botón a la inmunohistoquímica, mientras que los tumores DG presentan un patrón perinuclear difuso. En los últimos años se ha hecho evidente también la existencia de una población de tumores con un patrón mixto a la inmunhistoquímica para citoqueratina ${ }^{4}$. 
Esta clasificación del patrón granular tiene relevancia clínica, ya que según algunos reportes, los adenomas somatotropos EG podrían tener un comportamiento más agresivo que aquellos tumores DG y mixtos, los cuales se comportarían en forma similar ${ }^{4,11}$. Es así como los tumores EG tienden a presentarse a edades más tempranas, ser más grandes y más invasivos ${ }^{4-7}$. Respecto a la evolución postquirúrgica, los tumores EG presentan mayores tasas de resección incompleta y reoperación ${ }^{7,8}$. Esta clasificación tendría, además, un rol pronóstico en el tratamiento médico, ya que el patrón DG se asociaría a una respuesta bioquímica favorable al uso de ligandos del receptor de somatostatina (LRS) $)^{6,9-11,20,25}$.

Sin embargo, el pronóstico del tratamiento de los adenomas secretores de GH no solo está determinado por el patrón granular, sino también por una serie de características (por ejemplo, actividad bioquímica, tamaño, invasión) que tienden a cosegregarse $y$, por lo tanto, su importancia relativa es menos clara.

Los objetivos de este estudio son: a) comparar las características de presentación y los resultados quirúrgicos de pacientes con tumores secretores de GH según el patrón granular y b) definir factores pronósticos independientes del tratamiento quirúrgico en estos pacientes.

\section{Pacientes y Métodos}

\section{Pacientes}

Se realizó un estudio retrospectivo de los pacientes con adenoma somatotropo operados por dos neurocirujanos entre 1999 y 2014, en dos centros hospitalarios chilenos. El criterio de inclusión fue pacientes acromegálicos activos tratados primariamente con cirugía (resección tumoral transesfenoidal o transcraneal) y que no hubieran recibido tratamiento médico previo a la cirugía.

Los criterios de exclusión fueron: a) tumores somatotropos clínica y bioquímicamente inactivos y b) haber recibido LRS o radioterapia previo a la cirugía.

\section{Análisis bioquímico}

El diagnóstico de acromegalia se basó en: a) la presencia de niveles plasmáticos de IGF1 por sobre el rango de normalidad ajustado por edad y género, $\mathrm{o} b$ ) niveles plasmáticos de $\mathrm{GH}$ con un nadir $\geq 1 \mathrm{ng} / \mathrm{mL}$ durante un test de tolerancia a la glucosa ${ }^{12}$.

Las mediciones de IGF1 y de GH plasmáticas fueron hechas en ambos centros hospitalarios mediante inmunoensayo quimioluminiscente Immulite 2000. Siemens (Flanders, New Jersey, USA). Para IGF-1, la sensibilidad analítica es de $13,3 \mathrm{ng} / \mathrm{mL}$ con un coeficiente de variación analítico interensayo de 7,6\% para concentraciones de $56 \mathrm{ng} / \mathrm{mL}$ y de $3,9 \%$ para concentraciones de $200 \mathrm{ng} / \mathrm{mL}$. Para GH la sensibilidad analítica es de $0,01 \mathrm{ng} / \mathrm{mL}$ con un coeficiente de variación analítico interensayo de $6,9 \%$ para concentraciones de $3,6 \mathrm{ng} / \mathrm{mL}$ y de $8,2 \%$ para concentraciones de $9,4 \mathrm{ng} / \mathrm{mL}$. Se consideró el nadir de $\mathrm{GH}$ como el menor valor de GH obtenido en cinco muestras durante un test de sobrecarga con $75 \mathrm{~g}$ de glucosa. Se definió IGF1 indexada como la relación entre el valor de IGF1 plasmática preoperatoria de cada sujeto dividida por el valor superior del rango de normalidad para el mismo sujeto.

La medición de prolactina fue hecha por inmunoensayo quimioluminométrico (ADVIA Centaur o Immulite Siemens) y se consideró como hiperprolactinemia valores por sobre el nivel superior del rango de normalidad ajustados por edad y género.

La curación bioquímica postquirúrgica se definió por: a) niveles plasmáticos de IGF1 por debajo del valor máximo normal, o b) un nadir de $\mathrm{GH} \leq 0,4 \mathrm{ng} / \mathrm{mL}$ durante un test de sobrecarga con glucosa a los tres meses de la cirugía ${ }^{13}$. Se estableció la persistencia de enfermedad si al menos uno de estos criterios no se cumplía.

Este estudio fue aprobado por el Comité de Ética del Hospital Clínico de la Pontificia Universidad Católica de Chile.

\section{Análisis radiológico}

La interpretación de la resonancia magnética pre y postoperatoria fue hecha por dos neuroradiólogos independientes ( $\mathrm{IH}$ y RZ). El tumor fue clasificado como macroadenoma si medía más de $1 \mathrm{~cm}$ de diámetro mayor en la resonancia magnética (RM) preoperatoria. Se definió como invasión de seno cavernoso la presencia de un índice de Knosp $\geq 3$, esto es, extensión lateral a una línea imaginaria entre la tangentes laterales de las carótidas supra e intracavernosa ${ }^{14}$. 


\section{Histología e inmuohistoquímica}

Los tejidos resecados fueron fijados en formalina y embebidos en parafina. Se realizaron cortes finos, los que fueron utilizados para tinción con hematoxilina y eosina. Para el estudio inmunohistoquímico se usó el kit de detección universal DAB ultraView (Ventana Medical Systems, Tucson, Arizona, USA) y anticuerpos monoclonales para citoqueratina 8/18 (clon B22.1/B23.1) (Cell Marque de Millipore Sigma, Rocklin, California, USA) y anticuerpos policlonales contra $\mathrm{GH}$ y prolactina (Cell Marque de Millipore Sigma).

El patrón granular fue definido por un neuranatomopatólogo ( $R G$ ) como escasamente granular (EG) si la inmunohistoquímica para citoqueratina mostraba un patrón en botón, o densamente granular (DG) en aquellos con un patrón perinuclear o mixto de acuerdo a la actual clasificación de la Organización Mundial de la Salud (WHO) y estudios publicados previamente ${ }^{4,15}$.

\section{Análisis estadístico}

Se utilizó $\chi^{2}$ o test de Fisher para comparar variables dicotómicas y $\mathrm{T}$ test para comparar variables continuas. Se consideró un valor $\mathrm{p}<0,05$ como estadísticamente significativo.

$\mathrm{El}$ análisis de variables independientes que se asociaran a persistencia bioquímica de enfermedad se realizó a través de un modelo de regresión logística e incluyó solo aquellas variables que se asociaban significativamente en el análisis univariado. Todos los cálculos estadístico fueron hecho con el programa STATA ${ }^{\circledR}$ versión 12.0 de StataCorp LLC (College Station, Texas, USA).

\section{Resultados}

Se obtuvieron los registros clínicos de 85 tumores somatotropos que cumplían los criterios de inclusión y exclusión. En 3 de los 85 pacientes incluidos en el análisis no se obtuvieron datos del estado bioquímico de actividad postoperatoria (uno en grupo EG y dos en DG).

\section{Características basales del grupo total}

Las características clínicas, bioquímicas, radiológicas e inmunohistoquímicas basales del total de pacientes incluidos en el estudio se muestran en la Tabla 1.

Como se muestra, la edad promedio fue de 43 años, siendo $41 \%$ de los pacientes de sexo masculino. Del punto de vista bioquímico, la media de GH basal fue de $25,6 \mathrm{ng} / \mathrm{mL}$ y la IGF1 indexada de 2,9 y aproximadamente un tercio de los pacientes presentaba hiperprolactinemia asociado a la hipersecreción de GH. La RM preoperatoria mostraba macroadenomas en $68 \%$ de los pacientes e invasión de senos cavernosos en 39\%. Setenta y cuatro de los 85 pacientes incluidos en el estudio fueron clasificados como patrón DG (Figura 1) y 11 pacientes como patrón EG (Figura 2).

\section{Características según patrón inmunohistoquímico}

Dentro de las características basales no se observan diferencias significativas respecto de la edad de presentación o la distribución por género entre los grupos DG y EG (Tabla 2). Los tumores escasamente granulares eran bioquímicamente menos activos, con una menor GH basal que el grupo DG (13,8 vs $27,5 \mathrm{ng} / \mathrm{mL}$, respectivamente, $\mathrm{p}=0,008)$. La RM preoperatoria mostró una mayor frecuencia de macroadenomas en el grupo EG versus el grupo DG (100\% vs 63,5\%, respectivamente, $\mathrm{p}=0,014)$, así como también una mayor frecuencia de invasión de senos cavernosos $(72,7 \%$ vs $33,8 \%$, respectivamente, $\mathrm{p}=0,02)$.

Interesantemente, en la evolución postoperatoria se observó un significativo menor porcentaje de curación bioquímica en el grupo EG respecto del grupo DG ( $10 \%$ vs $50 \%, \mathrm{p}=0,02)$.

\section{Tabla 1. Características clínicas del grupo total de pacientes}

\begin{tabular}{|ll|}
\hline Variable 1 & Resultado \\
\hline Edad (años) & $43 \pm 14$ \\
\hline Masculino/Femenino & $35(41) / 50(59)$ \\
\hline IGF-1 indexada & $2,9 \pm 1,2$ \\
\hline GH basal (ng/mL) & $25,6 \pm 34$ \\
\hline GH nadir (ng/mL) & $19,8 \pm 27$ \\
\hline Hiperprolactinemia & $24(34)$ \\
\hline Macroadenoma/Microadenoma & $58(68) / 27(32)$ \\
Invasión de seno cavernoso & $32(39)$ \\
\hline Histología & $11(13)$ \\
Escasamente granular & $74(87)$ \\
\hline
\end{tabular}

Variables continuas expresadas como media $\pm \mathrm{DE}$; variables categóricas expresadas como $\mathrm{n}(\%)$. ' $\mathrm{E}$ I número de datos para cada variable no pudo ser obtenido para todos los pacientes. 

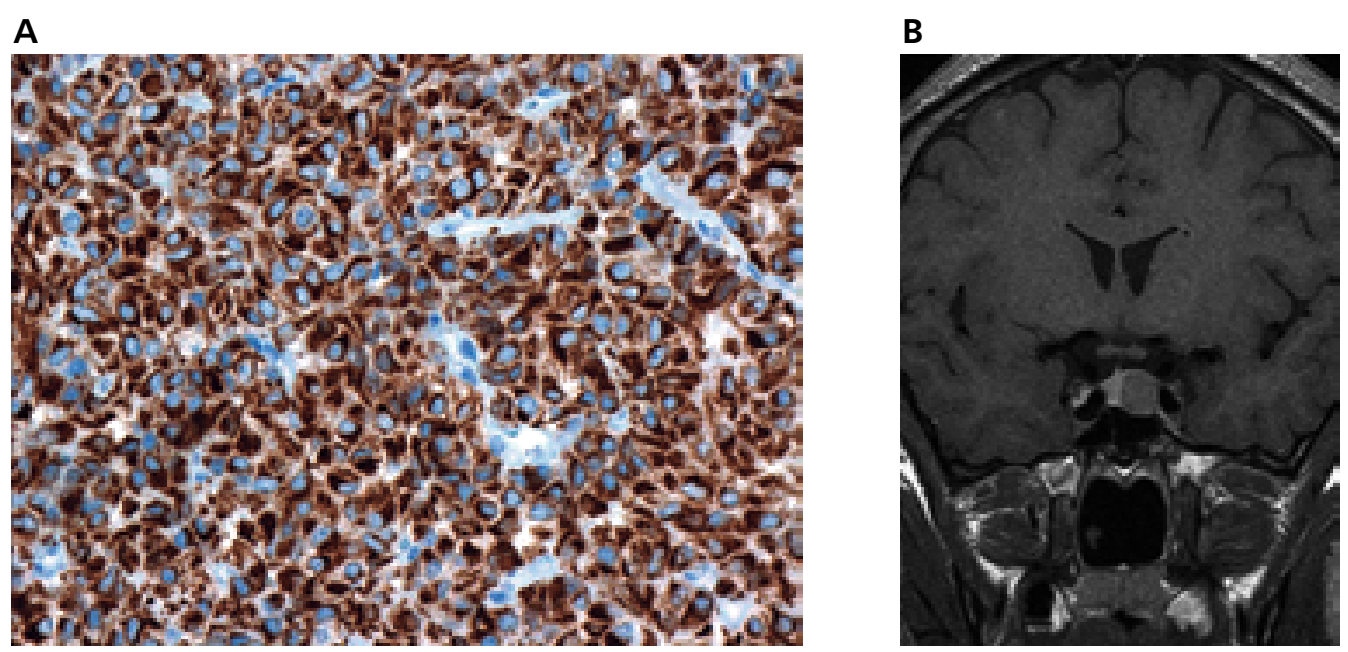

Figura 1. Caso representativo de un paciente acromegálico con tumor densamente granular. A) Inmunohistoquímica que muestra un patrón perinuclear difuso de filamentos de citoqueratina. B) Resonancia magnética que muestra un macroadenoma sin invasión de seno cavernoso.
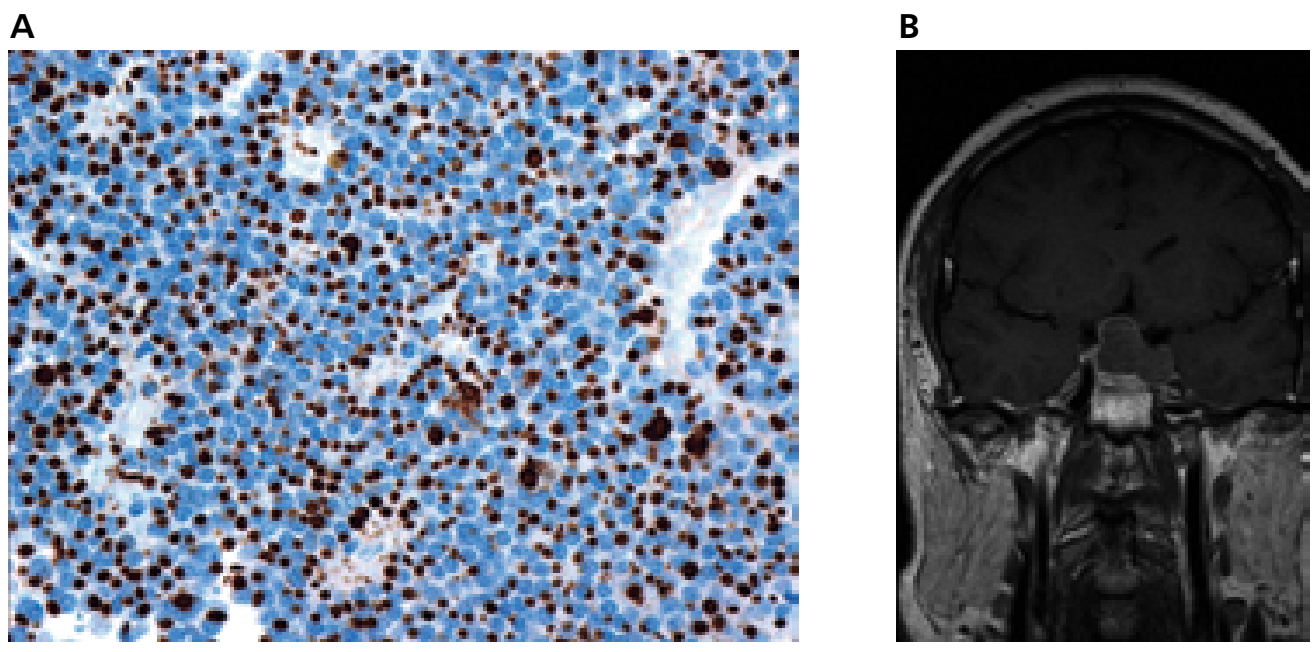

Figura 2. Caso representativo de un paciente acromegálico con tumor escasamente granular. A) Inmunohistoquímica que muestra un patrón en botón de filamentos de citoqueratina. B) Resonancia magnética que muestra un macroadenoma con extensión supraselar e invasión de seno cavernoso izquierdo.

\section{Predictores de persistencia de enfermedad}

De los ochenta y dos pacientes incluidos en el estudio que contaban con datos a los 3 meses postcirugía, $45(54,8 \%)$ presentaron persistencia bioquímica de enfermedad.

En el análisis univariado, los pacientes con persistencia bioquímica tenían una menor edad respecto de los pacientes curados (39 vs 49 años, $\mathrm{p}<0,01)$, una mayor GH basal (33,8 vs $17,2 \mathrm{ng} /$
$\mathrm{mL}, \mathrm{p}<0,05)$, mayores niveles de $\mathrm{GH}$ en el nadir $(28,7$ vs $11,7 \mathrm{ng} / \mathrm{mL}, \mathrm{p}<0,05)$, mayor frecuencia de macroadenomas $(77,7$ vs $54 \%, \mathrm{p}<0,05)$, de invasión de senos cavernosos $(65,1$ vs $8,3 \%$, $\mathrm{p}<0,01)$ y mayor frecuencia de patrón EG (20 vs $2,7 \%, \mathrm{p}<0,05)$. De estas características asociadas con persistencia de enfermedad, solo la edad $(\mathrm{OR}=0,93$; IC $0,87-0,99 ; \mathrm{p}<0,05)$ y la invasión de senos cavernosos $(\mathrm{OR}=21,7$; IC 2,07 - 227,61; 
Tabla 2. Características clínicas y resultado quirúrgico según patrón granular

\begin{tabular}{|lccc|}
\hline Variable & $\begin{array}{c}\text { DG } \\
(\mathbf{n = ~ 7 4 )}\end{array}$ & $\begin{array}{c}\text { EG } \\
\left(\mathbf{n}=\mathbf{1 1} \mathbf{1}^{\mathbf{1}}\right.\end{array}$ & Valor p \\
\hline Edad (años) & $44 \pm 14$ & $40 \pm 15$ & 0,219 \\
\hline Masculino/Femenino & $31(42) / 43(58)$ & $4(36) / 7(64)$ & 1,000 \\
IGF-1 indexada & $2,9 \pm 1,1$ & $3,2 \pm 1,7$ & 0,751 \\
GH basal (ng/mL) & $27,5 \pm 36$ & $13,8 \pm 11,5$ & 0,008 \\
GH nadir (ng/mL) & $20,6 \pm 28,2$ & $12,9 \pm 14,1$ & 0,256 \\
Hiperprolactinemia & $19(31)$ & $5(56)$ & 0,257 \\
Macroadenomas & $47(64)$ & $11(100)$ & 0,014 \\
\hline Invasión de seno cavernoso & $24(34)$ & $8(73)$ & 0,020 \\
\hline Curación bioquímica & $36(50)$ & $1(10)$ & 0,020 \\
\hline
\end{tabular}

Variables continuas expresadas como media $\pm \mathrm{DE}$; variables categóricas expresadas como $\mathrm{n}(\%)$. ${ }^{1} \mathrm{El}$ número de datos para cada variable no pudo ser obtenido para todos los pacientes.

Tabla 3. Resumen de las principales series publicadas

\begin{tabular}{|c|c|c|c|c|c|c|}
\hline Series & $\begin{array}{l}\text { Subtipo } \\
\text { histológico } \\
\text { (n) }\end{array}$ & $\begin{array}{l}\text { Edad } \\
\text { (años) }\end{array}$ & $\begin{array}{l}\text { Masculino/ } \\
\text { Femenino } \\
\text { (n) }\end{array}$ & $\underset{(\mathbf{n g} / \mathbf{m L})}{\mathbf{G H}}$ & $\begin{array}{l}\text { Invasión } \\
\qquad(\%)^{\dagger}\end{array}$ & Resultados de la intervención \\
\hline $\begin{array}{l}\text { 1992, } \\
\text { Bando }^{25}\end{array}$ & $\begin{array}{c}\mathrm{EG}=7 \\
\mathrm{DG}=13\end{array}$ & $\begin{array}{l}40 \\
47\end{array}$ & $\begin{array}{l}2 / 5 \\
4 / 9\end{array}$ & $\begin{array}{l}60 \\
33\end{array}$ & $\begin{array}{l}86 \\
31\end{array}$ & $\begin{array}{l}\text { EG mostró menor reducción de GH que } \\
\text { DG en test agudo de octreotide }\end{array}$ \\
\hline $\begin{array}{l}\text { 1993, } \\
\text { Yamada } 5\end{array}$ & $\begin{array}{c}E G=8 \\
D G=23\end{array}$ & $\begin{array}{l}37 \\
47\end{array}$ & $\begin{array}{c}1 / 7 \\
18 / 5\end{array}$ & $\begin{array}{l}71 \\
44\end{array}$ & $\begin{array}{l}88 \\
22\end{array}$ & $\begin{array}{l}\text { Menor curación quirúrgica en EG (13\%) } \\
\text { vs DG }(65 \%)\end{array}$ \\
\hline $\begin{array}{l}\text { 2001, } \\
\text { Mazal }^{8}\end{array}$ & $\begin{array}{l}\mathrm{EG}=29 \\
\mathrm{DG}=47\end{array}$ & $\begin{array}{l}39 \\
46\end{array}$ & $\begin{array}{c}9 / 20 \\
20 / 27\end{array}$ & ND & $\begin{array}{l}75 \\
26\end{array}$ & $\begin{array}{l}\text { EG mostró una mayor tasa de resecciones } \\
\text { incompletas ( } 34 \% \text { vs } 9 \% \text { y un menor } \\
\text { intervalo hasta re-operación que DG }\end{array}$ \\
\hline $\begin{array}{l}\text { 2008, } \\
\text { Obari }^{4}\end{array}$ & $\begin{array}{l}\mathrm{EG}=31 \\
\mathrm{DG}=47\end{array}$ & $\begin{array}{l}44 \\
50\end{array}$ & $\begin{array}{l}15 / 16 \\
26 / 21\end{array}$ & $\begin{array}{l}41 \\
59\end{array}$ & $\begin{array}{l}65 \\
38\end{array}$ & ND \\
\hline $\begin{array}{l}\text { 2010, } \\
\text { Bakhtiar }^{16}\end{array}$ & $\begin{array}{l}E G=30 \\
D G=83\end{array}$ & $\begin{array}{l}45 \\
51\end{array}$ & $\begin{array}{l}11 / 19 \\
40 / 43\end{array}$ & $\begin{array}{l}116 \\
46\end{array}$ & $\begin{array}{l}36 \\
36\end{array}$ & $\begin{array}{l}\text { Tasa de curación quirúrgica en EG } 42 \% \\
\text { vs DG } 63 \% \text { (estadísticamente no signi- } \\
\text { ficativo) }\end{array}$ \\
\hline $\begin{array}{l}\text { 2012, } \\
\text { Fougner }^{11}\end{array}$ & $\begin{array}{l}\mathrm{EG}=10 \\
\mathrm{DG}=31\end{array}$ & $\begin{array}{l}46 \\
56\end{array}$ & $\begin{array}{c}3 / 7 \\
13 / 18\end{array}$ & $\begin{array}{c}11 \\
9\end{array}$ & ND & $\begin{array}{l}\text { EG mostró menor reducción de GH que } \\
\text { DG en test agudo de octreotide }\end{array}$ \\
\hline $\begin{array}{l}\text { 2012, } \\
\text { Kato }^{20}\end{array}$ & $\begin{array}{l}E G=38 \\
D G=44\end{array}$ & $\begin{array}{l}41 \\
49\end{array}$ & $\begin{array}{l}17 / 21 \\
23 / 21\end{array}$ & $\begin{array}{l}76 \\
56\end{array}$ & $\begin{array}{l}57 \\
34\end{array}$ & $\begin{array}{l}\text { EG mostró menor reducción de GH que } \\
\text { DG en test agudo de octreotide }\end{array}$ \\
\hline $\begin{array}{l}\text { 2013, } \\
\text { Larkin }^{6}\end{array}$ & $\begin{array}{l}\mathrm{EG}=19 \\
\mathrm{DG}=23\end{array}$ & $\begin{array}{l}41 \\
52\end{array}$ & $\begin{array}{l}5 / 14 \\
14 / 9\end{array}$ & $\begin{array}{l}19 \\
31\end{array}$ & $\begin{array}{l}68 \\
21\end{array}$ & $\begin{array}{l}\text { EG mostró menor reducción de GH que } \\
\text { DG en test agudo de octreotide }\end{array}$ \\
\hline $\begin{array}{l}\text { 2013, } \\
\text { Brzana }^{9}\end{array}$ & $\begin{array}{l}\mathrm{EG}=14 \\
\mathrm{DG}=22\end{array}$ & $\begin{array}{l}42 \\
52\end{array}$ & $\begin{array}{c}3 / 11 \\
10 / 12\end{array}$ & ND & ND & $\begin{array}{l}\text { Sin diferencia en tasa de curación qui- } \\
\text { rúrgica, pero EG mostró una menor res- } \\
\text { puesta a LRS (67\%) que DG ( } 88 \%)\end{array}$ \\
\hline $\begin{array}{l}\text { 2015, } \\
\text { Kiseljak-Vassiliades }^{7}\end{array}$ & $\begin{array}{l}\mathrm{EG}=30 \\
\mathrm{DG}=71\end{array}$ & $\begin{array}{l}40 \\
50\end{array}$ & $\begin{array}{l}14 / 16 \\
37 / 34\end{array}$ & $\begin{array}{l}10 \\
12\end{array}$ & $\begin{array}{l}55 \\
36\end{array}$ & $\begin{array}{l}\text { Menor tasa de curación quirúrgica en EG } \\
(14 \%) \text { que en DG }(66 \%)\end{array}$ \\
\hline $\begin{array}{l}\text { 2019, } \\
\text { Valenzuela }\end{array}$ & $\begin{array}{l}E G=11 \\
D G=74\end{array}$ & $\begin{array}{l}40 \\
44\end{array}$ & $\begin{array}{c}4 / 7 \\
31 / 43\end{array}$ & $\begin{array}{l}14 \\
27\end{array}$ & $\begin{array}{l}73 \\
34\end{array}$ & $\begin{array}{l}\text { Menor tasa de curación quirúrgica en EG } \\
(10 \%) \text { que en DG }(50 \%)\end{array}$ \\
\hline
\end{tabular}

${ }^{\dagger}$ Adenomas invasores de acuerdo a sistemas de clasificación de Hardy o Knosp. GH = hormona de crecimiento; EG = tumores escasamente granulares; DG = tumores densamente granulares; ND = datos no disponibles; LRS = ligandos de receptor de somatostatina. 
$\mathrm{p}<0,01)$ se mantuvieron como variables independientes en el análisis multivariado de regresión logística.

\section{Discusión}

Este estudio retrospectivo, que incluyó 85 tumores somatotropos de pacientes con acromegalia activa operados, mostró que el grupo con patrón escasamente granular tenía diferencias significativas en la presentación bioquímica y radiológica respecto del grupo con patrón densamente granular.

A pesar de que la edad de los pacientes con adenomas EG fue menor que aquellos DG, esta diferencia no alcanzó significancia estadística, mientras que reportes previos han mostrado menor edad en los adenomas EG. Esto podría explicarse por un error estadístico tipo II, debido a un bajo número de tumores escasamente granulares en nuestra serie. Así también, no encontramos diferencias de género entre ambos tipos histológicos, lo cual está de acuerdo con algunos estudios $^{4,11,16}$, mientras que otros muestran un predominio de género femenino en los pacientes con tumores con patrón $\mathrm{EG}^{5,8}$. La Tabla 3 resume las características de los principales trabajos publicados previamente.

Del punto de vista bioquímico, este estudio mostró que los tumores EG tienen una menor $\mathrm{GH}$ basal. Las principales series publicadas previamente presentan resultados disímiles a este respecto; mientras en la serie de Obari ${ }^{4}$ los resultados fueron concordantes con los nuestros al presentar una menor GH basal en los tumores con patrón EG, la serie más grande publicada a la fecha por Bakhtiar ${ }^{16}$ no presentaba diferencias de actividad bioquímica entre los distintos patrones inmunohistoquímicos. La heterogeneidad entre los resultados de diferentes inmunoensayos para GH debida a variaciones en la calibración, especificidad de epítope y reconocimiento de diferentes isoformas de $\mathrm{GH}$ para el anticuerpo elegido ${ }^{17}$, podrían explicar la variabilidad de resultados en cuanto a la actividad secretora de tumores somatotropos con patrón EG. Algunos autores han postulado que la distribución en botón con agregados de citokeratina en cuerpos fibrosos produciría una disfunción de la célula adenomatosa, correlacionándose con menor cantidad de gránulos y menor expresión de RNA mensajero de $\mathrm{GH}^{23}$, lo que podría explicar la menor capacidad secretora de los tumores con patrón EG evidenciada en nuestro estudio.

Desde el punto de vista radiológico, los tumores con patrón EG tenían una mayor frecuencia de macroadenomas, lo cual parece ser una constante en la mayoría de las series publicadas ${ }^{4-6,8,11}$. La mayor tasa de invasión de seno cavernoso en nuestra serie está en concordancia con reportes previos. Parece claro en la actualidad que los tumores EG tendrían una menor expresion de E-cadherina ${ }^{16,18}$, relacionando la presencia de cuerpos fibrosos con la disfunción del sistema de adhesión celular lo que a su vez explicaría una mayor tendencia a la invasión.

Nuestro estudio demostró una significativa menor tasa de curación en los tumores con patrón EG respecto de aquellos con patrón DG (10 vs $50 \%, \mathrm{p}=0,02)$. El estudio publicado por Yama$\mathrm{da}^{5}$ fue el primero que reportó menores tasas de curación para tumores escasamente granulares con resultados muy similares a los nuestros (13\% vs $65 \%$ de curación para tumores escasamente vs densamente granular, respectivamente), lo que está en concordancia con la tendencia a menor curación reportada por Bakhtiar ${ }^{16}$, pero que no alcanzó significancia estadística ( $42 \%$ vs $60 \%$, $\mathrm{p}=0,076$ ). Las menores tasas de curación del grupo EG observadas en nuestro estudio respecto al de Bakhtiar ( $10 \%$ vs $42 \%$, respectivamente), podrían explicarse por una mayor frecuencia de invasión de seno cavernoso en nuestra serie de pacientes (72\% vs $35 \%$, respectivamente).

Interesantemente, nuestro estudio mostró que el patrón EG se asociaba a la persistencia bioquímica de enfermedad junto con otras variables previamente descritas ${ }^{19}$ como menor edad, mayores niveles de $\mathrm{GH}$, presencia de macroadenomas y de invasión de senos cavernosos. Sin embargo, muchas de estas variables mencionadas tienden a cosegregarse y su importancia relativa parece menos clara. Es así como en el presente estudio, solo la presencia de invasión del seno cavernoso y una menor edad se mantuvieron como variables independientes predictoras de la persistencia bioquímica de enfermedad en los pacientes operados de acromegalia. Dos estudios previos han publicado un análisis multivariado de factores pronósticos en pacientes con tumores secretores de $\mathrm{GH}$ tratados primariamente ${ }^{10} \mathrm{o}$ secundaria- 
mente ${ }^{26}$ con LRS, no incluyendo en este último el patrón granular dentro del análisis multivariado en el éxito de respuesta al tratamiento médico. Nuestro estudio es el primero en analizar variables pronósticas independientes en pacientes acromegálicos tratados primariamente con cirugía, lo que se condice con la realidad de la mayoría de los pacientes con diagnóstico de acromegalia en nuestro país.

En la última década, se han estudiado diversos parámetros moleculares como factores pronósticos de pacientes acromegálicos tratados quirúrgicamente o médicamente. Dentro de estos marcadores moleculares, solo una menor expresión del subtipo 2 del receptor de la somatostatina ${ }^{20,21} \mathrm{o}$ mutaciones del gen $\mathrm{AIP}^{22}$ tienen una evidencia sólida de asociación con un peor pronóstico. En este contexto y teniendo en cuenta que no contamos con un marcador ideal único, recientemente se ha propuesto un puntaje de riesgo que incluye varias variables clínicas, bioquímicas y radiológicas que también incluyen el patrón granular como un marcador de bajo costo ampliamente disponible ${ }^{19}$. De hecho, un estudio publicado más recientemente demostró que un patrón escasamente granular junto con el tamaño y la invasividad clasificaron correctamente a los pacientes acromegálicos que tenían un peor pronóstico ${ }^{24}$.

En suma, este estudio pone en evidencia que los tumores somatotropos con un patrón escasamente granular tienen una presentación más agresiva y menores tasas de curación que los tumores con patrón densamente granular. Sin embargo, la edad y la invasión de seno cavernoso son las únicas variables independientes preoperatorias que permitieron predecir el resultado post quirúrgico en nuestra serie de pacientes con acromegalia.

\section{Referencias}

1. Asa SL. The pathology of pituitary tumors. Endocrinol Metab Clin North Am 1999; 28 (1): 13-43.

2. Horvath E, Kovacs K. Pathology of Acromegaly. Neuroendocrinology 2006; 83 (3-4): 161-5.

3. Sano T, Ohshima T, Yamada S. Expression of glycoprotein hormones and intracytoplasmic distribution of cytokeratin in growth hormone-producing pituitary adenomas. Pathol Res Pract 1991; 187 (5): 530-3.

4. Obari A, Sano T, Ohyama K, Kudo E, Qian ZR, Yoneda A, et al. Clinicopathological features of growth hormo- ne-producing pituitary adenomas: difference among various types defined by cytokeratin distribution pattern including a transitional form. Endocr Pathol 2008; 19 (2): 82-91.

5. Yamada S, Alba T, Sano T, Kovacs K, Shishiba Y, Sawano S, et al. Growth hormone -producing pituitary adenomas: correlations between clinical characteristics and morphology. Neurosurgery 1993; 33 (1): 20-7.

6. Larkin S, Reddy R, Karavitaki N, Cudlip S, Wass J, Ansorge O. Granulation pattern, but not GSP or GHR mutation, is associated with clinical characteristics in somatostatin-naive patients with somatotroph adenomas. Eur J Endocrinol 2013; 168 (4): 491-9.

7. Kiseljak-Vassiliades K, Carlson NE, Borges MT, Kleinschmidt-DeMasters BK, Lillehei KO, Kerr JM, et al. Growth hormone tumor histological subtypes predict response to surgical and medical therapy. Endocrine 2015; 49 (1): 231-41.

8. Mazal PR, Czech T, Sedivy R Aichholzer M, Wanschitz J, Klupp N, et al. Prognostic relevance of intracytoplasmic cytokeratin pattern, hormone expresion profile and cell proliferation in pituitary adenomas of acromegalic patients. Clin Neuropathol 2001; 20 (4): 163-71.

9. Brzana J, Yedinak CG, Gultekin SH, Delashaw JB, Fleseriu M. Growth hormone granulation pattern and somatostatin receptor subtype $2 \mathrm{~A}$ correlate with postoperative somatostatin receptor ligand response in acromegaly: a large single center experience. Pituitary 2013; 16 (4): 490-8.

10. Bhayana S, Booth G, Asa SL, Kovacs K, Ezzat S. The implication of somatotroph adenoma phenotype to somatostatin analog responsiveness in acromegaly. J Clin Endocrinol Metab 2005; 90 (11): 6290-5.

11. Fougner SL, Casar-Borota O, Heck A, Berg JP, Bollerslev J. Adenoma granulation pattern correlates with clinical variables and effect of somatostatin analogue treatment in a large series of patients with acromegly. Clin Endocrinol (Oxf) 2012; 76 (1): 96-102.

12. Katznelson L, Laws E, Melmed S, Molitch ME, Murad $\mathrm{MH}, \mathrm{Utz}$ A, et al. Acromegaly: an endocrine society clinical practice guideline. J Clin Endocrinol Metab 2014; 99 (11): 3933-51.

13. Giustina A, Chanson P, Bronstein MD, Klibanski A, Lamberts S, Casanueva FF, et al. A consensus on criteria for cure of acromegaly. J Clin Endocrinol Metab 2010; 95 (7): 3141-8.

14. Knosp E, Steiner E, Kitz K, Matula C. Pituitary adenomas with invasion of the cavernous sinus space: a magnetic resonance imaging classification compared with surgical findings. Neurosurgery 1993; 33 (4): 610-8.

15. Kontogeorgos G. Growth hormone producing adenoma. 
En: DeLellis RA, Lioyd RV, Heitz PU, editores, Pathology and genetics of tumors of endocrine organs, IARC Press, Lyon 2004 p 14-9.

16. Bakhtiar Y, Hirano H, Arita K, Yunoue S, Fujio S, Tominaga $\mathrm{A}$, et al. Relationship between cytokeratin staining patterns and clinico-pathological features in somatotropinomae. Eur J Endocrinol 2010; 163 (4): 531-9.

17. Bidlingmaier M. Problems with GH assays and strategies toward standardization. Eur J Endocrinol 2008; 159 (Supl 1): S41-S4.

18. Xu B, Sano T, Yoshimoto K, Yamada S. Downregulation of E-cadherin and its undercoat proteins in pituitary growth hormone cell adenomas with prominent fibrous bodies. Endocr Pathol 2002; 13 (4): 341-51.

19. Fernández-Rodríguez E, Casanueva FF, Bernabeu I. Update on prognostic factors in acromegaly: is a risk score possible? Pituitary 2015; 18 (3): 431- 40.

20. Kato M, Inoshita N, Sugiyama T, Tani Y, Shichiri M, Sano T, et al. Differential expression of genes related to drug responsiveness between sparsely and densely granulated somatotroph adenomas. Endocrine J 2012; 59 (3): 221-8.

21. Mayr B, Buslei R, Theodoropoulou M, Buchfelder M, Schöfl C. Molecular and functional properties of densely and sparsely granulated GH-producing pituitary adenomas. Eur J Endocrinol 2013; 169 (4): 391-400.
22. Daly A, Tichomirowa M, Petrossians P, Heliövaara E, Jaffrain-Rea ML, Barlier A, et al. Clinical characteristics and therapeutic responses in patients with germ-line AIP mutations and pituitary adenomas: an international collaborative study. J Clin Endocrinol Metab 2010; 95 (11): E373-83.

23. Yamada S, Takahashi M, Hara M, Sano T, Aiba T, Shishiba Y, et al. Growth hormone and prolactin gene expression in human densely and sparsely granulated somatotroph adenomas by in situ hybridization with digoxigenin-labeled probes. Diagn Mol Pathol 1994; 3 (1): 46-52.

24. Cuevas-Ramos D, Carmichael JD, Cooper O, Bonert VS, Gertych A, Mamelak AN, et al. A structural and functional acromegaly classification. J Clin Endocrinol Metab 2015; 100 (1): 122-31.

25. Bando H, Sano T, Ohshima T, Zhang CY, Yamasaki R, Matsumoto K, et al. Differences in pathological findings and growth hormone responses in patients with growth hormone-producing pituitary adenoma. Endocrinol Jpn 1992; 39 (4): 355-63.

26. Kasuki L, Wildemberg LE, Neto LV, Marcondes J, Takiya CM, Gadelha MR. Ki-67 is a predictor of acromegaly control with octreotide LAR independent of SSTR2 status and relates to cytokeratin pattern. Eur J Endocrinol 2013; 169 (2): 217-23. 